PROCEEDINGS OF THE

AMERICAN MATHEMATICAL SOCIETY

Volume 135, Number 8, August 2007, Pages 2661-2664

S 0002-9939(07)08782-5

Article electronically published on March 30, 2007

\title{
EMBEDDINGS OF $n$-DIMENSIONAL SEPARABLE METRIC SPACES INTO THE PRODUCT OF SIERPIŃSKI CURVES
}

\author{
DARIA MICHALIK
}

(Communicated by Alexander N. Dranishnikov)

\begin{abstract}
We give a short proof of the following fact: the set of embeddings of any $n$-dimensional separable metric space $X$ into a certain $n$-dimensional subset of the $(n+1)$-product of Sierpiński curves $\Sigma$ is residual in $C\left(X, \Sigma^{n+1}\right)$.
\end{abstract}

\section{INTRODUCTION AND NOTATION}

In a Sierpiński curve we can specify the 0-dimensional subset of a "rational" points. In [5, Ivanšić and Milutinović proved that the $(n+1)$-product of Sierpiński curves, with points whose coordinates are all rational removed, is a universal space for $n$-dimensional metric separable spaces.

In this paper we present a new short proof of the last result. The proof is similar in spirit to proof of Theorem 1.1 in [6]. Sternfeld proved in his paper that any $n$-dimensional compact metric space may be embedded in the $(n+1)$-product of dendrites $D$. He also showed that the set of the basic embeddings is dense in $C\left(X, D^{n+1}\right)$. However, it is worth pointing out that concerned in $[6]$ is the fact that dendrites are ARs. Since a Sierpiński curve has not a good extension property, we use Lemma 3 to approximate it by ANRs. We also use in our proof the idea of the disjoint disk property that has been used in the topology of infinite- or finitedimensional manifolds (cf. 7]).

All maps in this paper are continuous. Maps $f, g: X \rightarrow Y$ are said to be $\varepsilon$-near if $\sup _{x \in X} \operatorname{dist}(f(x), g(x))<\varepsilon$. A map $f: X \rightarrow Y$ is an $\varepsilon$-map if each point $y \in Y$ has an open neighbourhood $V_{y}$ such that $\operatorname{diam}\left(f^{-1}\left(V_{y}\right)\right)<\varepsilon$. We denote by $B(x, r)$ the open ball with centre $x$ and radius $r$.

We shall use the following proposition (it is an easy application of Eilenberg's Theorem; see [1])

Proposition 1. Let $X$ be a compact metric space and $Y$ be a metric ANR. Then for each map $f: X \rightarrow Y$ and each $\varepsilon>0$ there exists $a \delta>0$ such that for each surjective $\delta$-map $p: X \rightarrow X^{\prime}$ there exists a map $q: X^{\prime} \rightarrow Y$ such that $\operatorname{dist}(f, q \circ p)<\varepsilon$.

Received by the editors March 21, 2005 and, in revised form, April 28, 2005.

2000 Mathematics Subject Classification. Primary 54F45, 14C55; Secondary 54C25, 54F50.

Key words and phrases. Covering dimension, Sierpiński curve, embedding, ANR.

(C)2007 American Mathematical Society Reverts to public domain 28 years from publication 


\section{MAIN THEOREM}

Let us recall the construction of the triangular Sierpiński curve. Consider the homotheties $\varphi_{i}: \mathbb{R}^{3} \rightarrow \mathbb{R}^{3}$ with scale $\frac{1}{2}$ and centres $e_{i}$, where $e_{1}=(1,0,0), e_{2}=$ $(0,1,0), e_{3}=(0,0,1)$. With $\Sigma=\operatorname{conv}\left(e_{1}, e_{2}, e_{3}\right)$ we let

$$
\Sigma_{m}:=\bigcup_{\lambda_{1}, \ldots, \lambda_{m} \in \Lambda} \varphi_{\lambda_{1}} \circ \cdots \circ \varphi_{\lambda_{m}}(\Sigma) \text {, where } \Lambda=\{1,2,3\} \text {, and } \Sigma(3):=\bigcap_{m \in \mathbb{N}} \Sigma_{m} \text {. }
$$

We also let $\Sigma_{n}(3):=\Sigma \backslash$ Int $\Sigma_{n}$. Clearly, $\Sigma_{n}(3)$ is a graph and hence an ANR, for each $n \in \mathbb{N}$.

We call the vertices of triangles obtained in the construction of $\Sigma(3)$ rational points, and other points of $\Sigma(3)$ irrational points. Let

$$
L_{n}(3):=\left\{x \in \Sigma(3)^{n+1}: \text { at least one coordinate of } x \text { is irrational }\right\} .
$$

Theorem 2. Suppose that $X$ is a metric separable space. If $\operatorname{dim}(X) \leq n$, then the set of embeddings of $X$ in $L_{n}(3)$ is dense in the space $C\left(X, \Sigma(3)^{n+1}\right)$.

The original proof [5] of this result depended on complex arguments involving brick decompositions. Instead, here we use a simple property of compact metric spaces and the fact that a Sierpiński curve can be approximated by the graphs $\Sigma_{n}(3):$

Lemma 3. For each $\varepsilon>0$ there exists a natural number $k$ and a retraction $r_{k}$ of $\Sigma(3)$ onto $\Sigma_{k}(3)$ such that $d\left(r_{k}, i d_{\Sigma(3)}\right)<\varepsilon$.

Proof. Let $k$ be such that $\frac{1}{2^{k}}<\varepsilon$. For each $\lambda=\left(\lambda_{1}, \ldots, \lambda_{k}\right) \in \Lambda^{k}$ the Sierpiński curve $\Sigma(3)$ intersects the simplex $S_{\lambda}=\varphi_{\lambda_{1}} \circ \cdots \circ \varphi_{\lambda_{k}}(\Sigma)$ along a set containing the boundary of $\operatorname{Bd}\left(S_{\lambda}\right)$ but different from $S_{\lambda}$. Therefore $\Sigma(3) \cap S_{\lambda}$ may be retracted onto $\operatorname{Bd}\left(S_{\lambda}\right)$. The union of these retractions, taken over all $\lambda \in \Lambda^{k}$, does the job.

Lemma 4. Let $X$ be a compact metric space and $f: X \rightarrow \Sigma(3)^{n+1}$ be a map. Then for each $\varepsilon>0$ and for each pair of disjoint compact sets $A, B \subset X$ such that $\operatorname{dim} A \leq n$, there exists a map $f_{\varepsilon}: X \rightarrow \Sigma(3)^{n+1}$ such that $\operatorname{dist}\left(f, f_{\varepsilon}\right)<\varepsilon$, $f_{\varepsilon}(A) \cap f_{\varepsilon}(B)=\emptyset$ and $f_{\varepsilon}(A) \subset L_{n}(3)$.

Proof. The proof is by induction on $n$. First let $n=0$. We fix $\varepsilon>0$ and take $k$ so large that the retraction $r_{k}: \Sigma(3) \rightarrow \Sigma_{k}(3)$ satisfies $\operatorname{dist}\left(\operatorname{id}_{\Sigma(3)}, r_{k}\right)<\varepsilon / 3$. We also cover $A$ by finitely many disjoint $\delta$-small compact sets, where $\delta<\operatorname{dist}(A, B)$, and denote by $X^{\prime}$ the space obtained from $X$ by squeezing each of them to a point. Let us observe that by [3] (Theorem 4.4.15) $X^{\prime}$ is a metrizable space. By Proposition 1 . for $\delta$ small enough the map $r_{k} \circ f$ is $\varepsilon / 3$-close to the composition of the projection $p: X \rightarrow X^{\prime}$ and of a map $f^{\prime}: X^{\prime} \rightarrow \Sigma_{k}(3) \subset \Sigma(3)$. Thus, by replacing $f$ by $f^{\prime}$, and $A$ and $B$ by $p(A)$ and $p(B)$, respectively, we may assume that the set $A$ is finite, and by treating each of its points individually - that $A$ has only one point, which we denote $a$. We assume these arrangements have been made; in particular, $\operatorname{Im}(f) \subset \Sigma_{k}(3)$.

Let $B(a, \eta)$ be a ball in $X$ centred at $a, W=X / \operatorname{Bd} B(a, \eta)$ be the decomposition space, obtained by squeezing $\operatorname{Bd} B(a, \eta)$ to a point, and let $p: X \rightarrow W$ be the projection. By taking $\eta$ small enough we ensure that $\operatorname{diam} f(B(a, \eta))<\varepsilon / 3$ and there exists a map $q: W \rightarrow \Sigma_{k}(3) \subset \Sigma(3)$ such that $q \circ p$ is $\varepsilon / 3$-near to $f$. (We use Proposition 1]) 
Let us fix $b \in \operatorname{Bd}(B(a, \eta))$. There exists an $\varepsilon / 3$-short arc $J$ in $\Sigma(3)$ joining $q(p(b))$ with an irrational point $c \in \Sigma(3) \backslash \Sigma_{k}(3)$. Let $h: W \rightarrow \Sigma(3)$ be a map such that $h(x)=q(x)$ for $x \in W \backslash p(B(a, \eta))$ and $h(p(a))=c$. The arc $J$ is an AR so we can extend the map $h$ to the whole space $W$ so that $h(p(B(a, \eta)) \subset J$.

Let us define $f_{\varepsilon}=h \circ p$. If $x \in X \backslash B(a, \eta)$, then $f_{\varepsilon}(x)=q \circ p(x)$ and hence $d\left(f_{\varepsilon}(x), f(x)\right)<\varepsilon / 3$, while for $x \in B(a, \eta)$ we have $f(x), f_{\varepsilon}(x) \in J$ and $f(b), f(x) \in$ $f(B(a, \eta))$, whence

$$
d\left(f_{\varepsilon}(x), f(x)\right) \leq d\left(f_{\varepsilon}(x), f_{\varepsilon}(b)\right)+d\left(f_{\varepsilon}(b), f(b)\right)+d(f(b), f(x))<3 \cdot \varepsilon / 3
$$

because $\operatorname{diam} J, \operatorname{diam} f(B(a, \eta))<\varepsilon / 3$. Thus $d\left(f, f_{\varepsilon}\right)<\varepsilon$ and since $f_{\varepsilon}(a) \in \Sigma(3) \backslash$ $\Sigma_{k}(3)$ and $f_{\varepsilon}(B) \subset \Sigma_{k}(3)$ the assertion of the lemma is true if $n=0$.

Assume now that the lemma holds true if $\operatorname{dim} A \leq n-1$. From the Baire Theorem and from the fact that $L_{0}(3)$ is of $G_{\delta}$ type we conclude that

$$
\text { the lemma is true if } A \text { is } \sigma \text { - compact and } \operatorname{dim} A \leq n-1 \text {. }
$$

Now, given $f=\left(f_{1}, f_{2}\right): X \rightarrow \Sigma(3)^{n} \times \Sigma(3)$ and a compact set $A \subset X$ with $\operatorname{dim} A \leq n$, we can represent $A$ as $A=Y \cup Z$, where $\operatorname{dim} Y \leq n-1, \operatorname{dim} Z \leq 0$ and $Z$ is $\sigma$-compact. (See $\left[2, \S 1.5\right.$.) By $(*)$ we can assume that $f_{2}(Z) \cap f_{2}(B)=\emptyset$ and $f_{2}(Z) \subset L_{0}(3)$. The set $Y^{\prime}=\left(\left.f_{2}\right|_{A}\right)^{-1}\left(f_{2}(B) \cup\left(\Sigma(3) \backslash L_{0}(3)\right)\right)$ is disjoint from $Z, n$-1-dimensional, and $\sigma$-compact (for $L_{0}(3)$ is of type $G_{\delta}$ ). Thus by (*) there exists a map $f_{1}^{\varepsilon}: X \rightarrow \Sigma(3)^{n} \varepsilon$-near to $f_{1}$ such that $f_{1}^{\varepsilon}\left(Y^{\prime}\right) \subset L_{n-1}(3)$ and $f_{1}^{\varepsilon}\left(Y^{\prime}\right) \cap f_{1}^{\varepsilon}(B)=\emptyset$. We take $f_{\varepsilon}(x)=\left(f_{1}^{\varepsilon}(x), f_{2}(x)\right)$ to complete the proof.

Proof of Theorem 2. Let $f \in C\left(X, \Sigma(3)^{n+1}\right)$. By [4 there exists a dimension preserving compactification $X^{*}$ of the space $X$ such that the map $f: X \rightarrow \Sigma(3)^{n+1}$ can be extended to $f^{*}: X^{*} \rightarrow \Sigma(3)^{n+1}$. Hence, without loss of generality, we may assume that $X$ is compact.

Let $\mathcal{B}$ be a countable family of closed subsets of $X$ whose interiors are a base of the topology of $X$. Let us observe that

$$
\begin{aligned}
\{h \in & \left.C\left(X, \Sigma(3)^{n+1}\right): h \text { is an embedding }\right\} \\
& =\bigcap_{\substack{A, B \in \mathcal{B} \\
A \cap B=\emptyset}}\left\{f \in C\left(X, \Sigma(3)^{n+1}\right): f(A) \cap f(B)=\emptyset\right\} .
\end{aligned}
$$

Fix $A, B \in \mathcal{B}$ such that $A \cap B=\emptyset$. By Lemma 4 the set $\left\{f \in C\left(X, \Sigma(3)^{n+1}\right)\right.$ : $f(A) \cap f(B)=\emptyset\}$ is dense in $C\left(X, \Sigma(3)^{n+1}\right)$. Also by Lemma 4 (with $A=X$ and $B=\emptyset)$ the set $\left\{f \in C\left(X, \Sigma(3)^{n+1}\right): f(X) \subset L_{n}(3)\right\}$ is dense in $C\left(X, \Sigma(3)^{n+1}\right)$. The sets $\left\{f \in C\left(X, \Sigma(3)^{n+1}\right): f(X) \subset L_{n}(3)\right\}$ and $\bigcap_{\substack{A, B \in \mathcal{B} \\ A \cap B=\emptyset}}\left\{f \in C\left(X, \Sigma(3)^{n+1}\right)\right.$ : $f(A) \cap f(B)=\emptyset\}$ are obviously of type $G_{\delta}$. Now the proof is completed by an application of Baire's Theorem.

Remark 5. The following property of a Sierpiński curve can be proved analogously to Lemma 4:

Let $F$ be a $\sigma$-closed 0 -dimensional subset of a compact metric space $X$. Then $\left\{f \in C(X, \Sigma(3)): f^{-1} f(x)=\{x\}\right.$ for all $x \in F$ and $f(F)$ contains no rational points $\}$ is a dense $G_{\delta}$-set in $C(X, \Sigma(3))$.

This fact is similar to Theorem 1.1 in [6] that is a key result of Sternfeld's paper. Using this property of a Sierpiński curve and the argument that the set of trivial fibers of map $f$ is $G_{\delta}$-set we can construct an embedding into $L_{n}(3)$. This way of construction is analogous to Sternfeld's methods in [6]. 
Remark 6. In [6], Sternfeld noted that in the case of embeddings into $(n+1)$ product of dendrites the last space in that product can be replaced by an interval. The same can be done also for the product of Sierpiński curves.

\section{ACKNOWLEDGMENTS}

I would like to thank Professor H. Toruńczyk for suggesting the problem and for many hours of conversation. I am also grateful to the referee for drawing my attention to Sternfeld's paper and suggesting Remark 5 .

\section{REFERENCES}

1. S. Eilenberg, Sur les transformations à petites tranches, Fund. Math. 30 (1938), 92-95.

2. R. Engelking, Theory of dimensions finite and infinite, Heldermann Verlag, Lemgo, 1995. MR.1363947 (97j:54033)

3. R. Engelking, General topology, Heldermann Verlag, Berlin, 1989. MR.1039321(91c:54001)

4. A. B. Forge, Dimension preserving compactifications allowing extensions of continuous functions, Duke Math. Journ. 28 (1961), 625-627. MRMR0167955 (29:5220)

5. I. Ivanšić, U. Milutinović, A Universal Separable Metric Space Based on Triangular Sierpinski Curve, Topology Appl. 120 (2002), 237-271. MR1895494 (2003a:54039)

6. Y. Sternfeld, Mappings in dendrites and dimension, Houston Journal of Math. 19 (1993), 483-497. MR.1242434 (94j:54017)

7. H. Toruńczyk, Finite-to-one restrictions of continuous functions, 2, General Topology and its Relations to Modern Analysis and Algebra VI, Proc. Sixth. Prague Topological Symposium 1986, 589-599. MR0952641(89j:57009)

Institute of Mathematics, Polish Academy of Sciences, P.O. Box 21 Śniadeckich 8 00-956 Warszawa, Poland

E-mail address: daria@impan.gov.pl 